\title{
Dental injury with alveolar bone fracture during mechanical ventilation: a case report
}

\author{
Mariko Yamakawa ${ }^{1}$, Toshinide Sato $^{2}$, Tetsuro Mitsuse $^{3}$, and Tatsuhiko Kawaguchi ${ }^{1}$ \\ ${ }^{1}$ Department of Dentistry, Kumamoto City Hospital, 1-1-60 Koto, Kumamoto 862-8505, Japan \\ ${ }^{2}$ Intensive Care Unit, Kumamoto City Hospital, Kumamoto, Japan \\ ${ }^{3}$ Surgical Center, Kumamoto City Hospital, Kumamoto, Japan
}

Key words: Complication, Dental accident, Mechanical ventilation

\section{Introduction}

Traumatic injuries to the teeth sometimes occur during endotracheal intubation under general anesthesia [1,2]. However, there are few reports of traumatic injuries affecting the alveolar bone in patients during respiratory management in the intensive care unit (ICU). We report a dental injury accompanied by an alveolar bone fracture at the lower anterior site, as well as subluxation of the upper and lower incisors caused by vigorous biting on a latex bite block during mechanical ventilation.

\section{Case report}

A 49-year-old man, $155 \mathrm{~cm}$ tall and weighing $55 \mathrm{~kg}$, was referred to our hospital because of exacerbated cardiac dysfunction. His medical history included a myocardial infarction with cardiac arrest 5 years before, and a cerebral infarction 3 years prior to arrival. Four days after admission, the patient was moved to the ICU because of a progressive hepatic coma. Before he arrived in the ICU, an orotracheal tube was inserted. Cheyne-Stokes breathing was observed under pressure support ventilation using a Servo $900 \mathrm{C}$ Ventilator. The results of the arterial blood gas analyses on $\mathrm{FiO}_{2} 1.0$, were as follows; pH 7.63, $\mathrm{PaO}_{2} 199 \mathrm{mmHg}, \mathrm{PaCO}_{2} 28.9 \mathrm{mmHg}$, and base excess $9.4 \mathrm{mmol} \cdot 1^{-1}$. His pulse was regular, with slightly

Address correspondence to: M. Yamakawa

Received for publication on June 7, 1999; accepted on October 6, 1999 weak tension at a rate of 92 beats $\mathrm{min}^{-1}$. His blood pressure was $146 / 86 \mathrm{mmHg}$ after administration of $5.1 \mu \mathrm{g} \cdot \mathrm{kg}^{-1} \cdot \mathrm{min}^{-1}$ of dopamine. His axillary temperature was $38.1^{\circ} \mathrm{C}$. The Glasgow coma scale showed 7; eyeopening 2 , verbal response 1 , and motor response 4 . His pupils were isocoric, round, highly reactive to light, and undilated. Cranial computed tomography revealed no pathological region except at the old cerebral infarction. There was significant jaundice on his skin and bulbar conjunctiva. The abnormal laboratory findings were as follows: total bilirubin, $3.4 \mathrm{mg} \cdot \mathrm{dl}^{-1}$; aspartate aminotransferase, $1552 \mathrm{IU} \cdot \mathrm{l}^{-1}$; alanine aminotransferase, $1708 \mathrm{IU} \cdot \mathrm{l}^{-1}$; lactate dehydrogenase, $1836 \mathrm{IU} \cdot \mathrm{l}^{-1}$; alkaline phosphatase, $290 \mathrm{IU} \cdot \mathrm{l}^{-1}$; international normalized ratio (INR), 4.7; blood urea nitrogen, $40.9 \mathrm{mg} \cdot \mathrm{dl}^{-1}$; serum sodium, $156 \mathrm{mEq} \cdot \mathrm{l}^{-1}$; and serum chloride, $114 \mathrm{mEq} \cdot 1^{-1}$. Hepatic dysfunction was partly eased with a fresh frozen plasma infusion. Cardiac function was supported with continuous administration of dopamine.

The endotracheal tube was positioned at a depth of $23 \mathrm{~cm}$ on the right maxillary central incisor using adhesive tape. A latex bite block was inserted between the upper and the lower teeth to avoid involuntary biting of the tube. The patient often bit on the bite block in response to endotracheal suctioning or oral cavity cleaning. At the first oral examination in the ICU on day 1 , no significant dental findings were noted other than an absence of left mandibular molars. On day 3, a number of anterior teeth in contact with the bite block were noted to be mobile and elongated. A closer examination revealed incomplete dislocation of these teeth and bleeding from the gingiva (Fig. 1). Moreover, moderate mobility of the alveolar bone was detected. These findings suggested that the alveolar bone sockets were not intact and were probably fractured. However, portable radiography showed only an enlargement of the periodontal ligament space for the incisors, but did not show a fracture line. Dental treatment was performed 


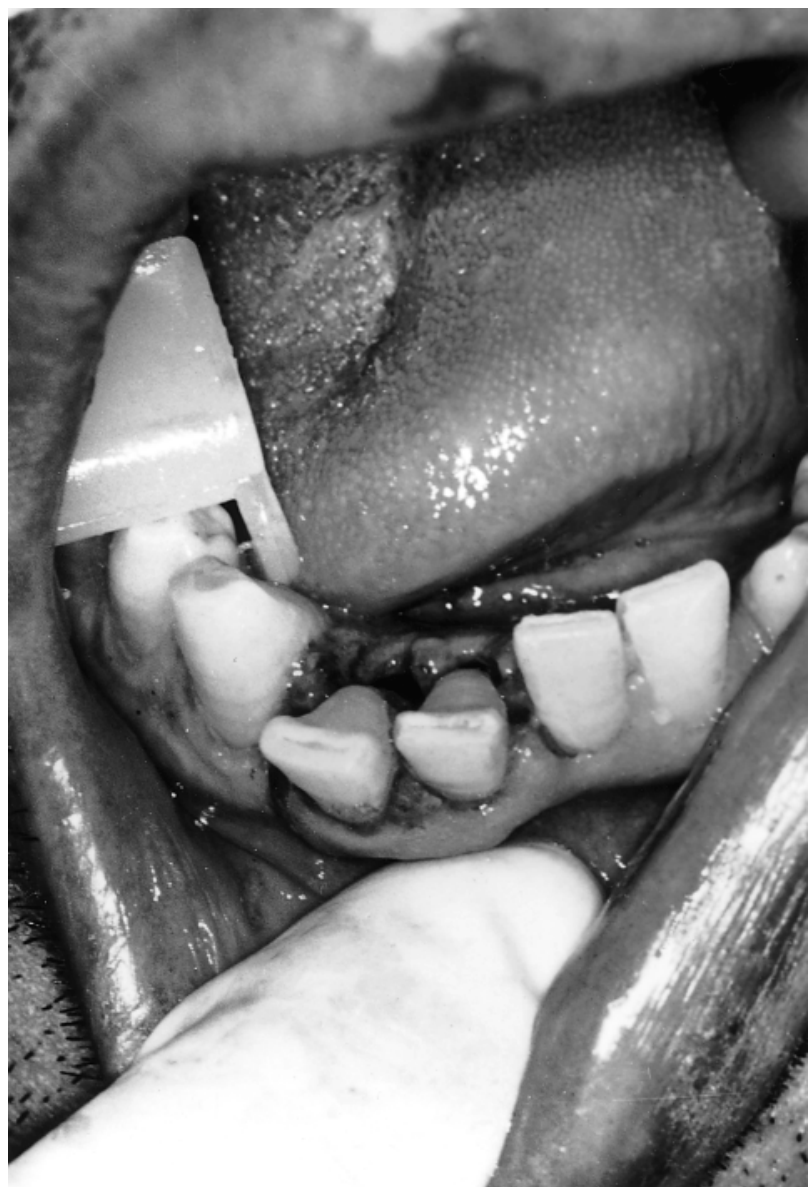

Fig. 1. Incomplete dislocation of lower anterior teeth

under intravenous anesthesia using neuroleptics, analgesics, and muscle relaxants. After the dislocated teeth had been reimplanted into their sockets, a splint was applied from the left to the right canines to stabilize the reimplanted teeth. We used a $0.7-\mathrm{mm}$ stainless steel orthodontic wire and acid etch-composite resin as a resin wire splint (Fig. 2). The endotracheal tube and the bite block were repositioned on the posterior site of the dentition. Sufficient doses of sedatives including midazolam and buprenorphine were useful to prevent involuntary biting. He gradually regained consciousness as his liver function improved. The endotracheal tube was removed on day 15 , and the patient was discharged from the ICU on day 19. Although the undesirable symptoms had almost disappeared from the anterior teeth by the day of discharge, the splint was left in place for another four weeks. Nine days after discharge, a careful examination was conducted by dental radiography. The examination identified an enlargement of the periodontal ligament space and a fracture line of the alveolar bone, indicating that the patient had had an alveolar bone fracture.

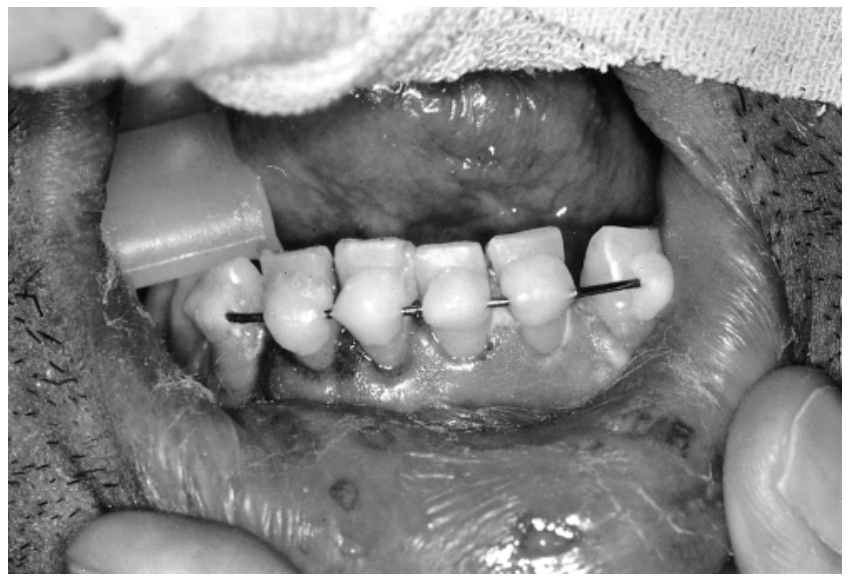

Fig. 2. A splint applied from the left to the right canines to stabilize the reimplanted teeth

\section{Discussion}

Alveolar bone fracture is usually caused by violent trauma, such as traffic and sports accidents and fights [3]. Although tooth injuries during endotracheal intubation are common [1,2], few articles have reported alveolar bone fractures during mechanical ventilation. In this patient, an alveolar bone fracture with tooth subluxation from the sockets probably was caused by vigorous biting on the latex bite block. In our ICU, endotracheal intubation is usually performed orotracheally. The tube is fixed at a short distance from the lip angle to prevent pressure injury of the lip and skin. In this patient, a latex bite block was exceptionally necessary to protect the endotracheal tube from involuntary biting while he was comatose. This excessive external force was most likely transferred to the right upper central incisor and lower central and lateral incisors directly, thus causing a severe injury. In the literature [4,5], tooth damage has been reported from such vigorous biting upon an in situ oropharyngeal airway during masseter muscle spasms when volatile agents such as halothane were used as a general anesthetic. However, there was no damage to the alveolar bone in these patients.

There were visible signs of an enlargement of the periodontal ligament space of the anterior teeth on portable X-ray film. Although no distinct fracture line was radiographically visible, the moderate mobility of the alveolar bone during palpation and the bleeding from the damaged gingiva were strong evidence of tooth subluxation with alveolar bone fracture. The diagnosis of an alveolar bone fracture was convincing.

Immediate steps should be taken to minimize permanent damage. Loose teeth and fractured bone should be manually replaced into their original position. A splint apparatus, such as a hard acrylic splint, wire and acrylic 


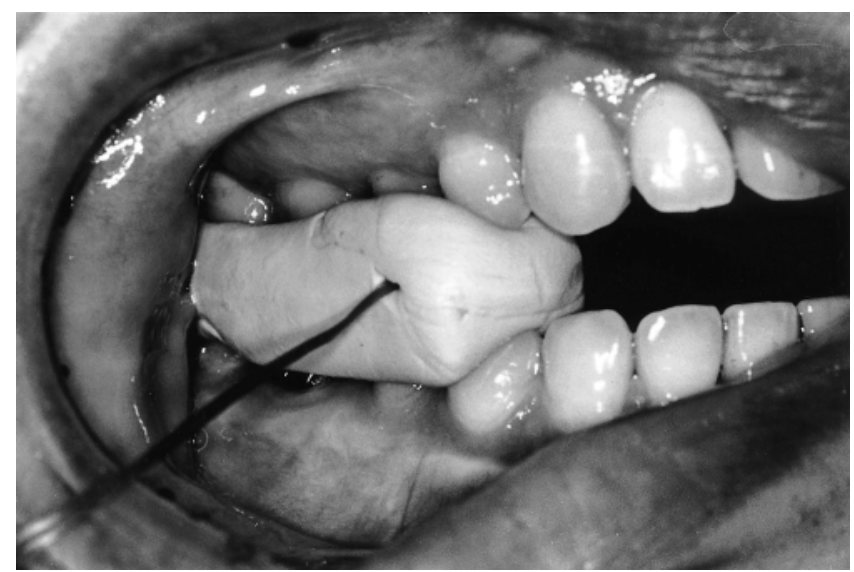

Fig. 3. A silicone rubber bite block placed between dentitions

splints, or custom-fitted mouth guards, is required to stabilize the injured tissues. If mucosal lacerations and/ or exposure to pulp are found, suture and/or endodontic treatment are necessary. Surgery is required if there is wide dislocation between the fractured fragments that involve a large number of teeth. In this patient, we did not notice any excessive dislocation between the segments or any tooth root fracture. Therefore, we could nonsurgically put the bone and teeth back into their original positions. We treated this patient quickly and adequately, securing a good outcome.

After the reduction and fixation of the lesions, the tube and bite block were moved to the posterior site to reduce and disperse bite pressure, thus avoiding recurrence of injury during the healing period. Molars with multiple roots are less vulnerable to external force than incisors with single roots unless there is severe periodontal disease. Therefore, fixation of the bite block to a posterior site of the dentition is recommended be- cause the teeth and the supporting structures are spared traumatic injuries caused by unusual amounts of nondirected masticatory force. We also recommend that patients undergoing mechanical ventilation under endotracheal intubation have a dental check whenever possible to prevent self-injury.

Protective appliances are used to prevent dental accidents during general anesthesia [6,7]. There is, however, some difficulty in providing such appliances for patients in respiratory management in the ICU. After the present case, we designed a custom-fitted bite block made of silicone rubber, a material of dental modeling. The silicone rubber bite block could be easily made and placed at the bedside (Fig. 3). We have successfully used this instrument for dental protection and oral care in critically ill, unconscious patients. We hope that techniques for accident prevention will remain a subject of discussion so that further innovations can be made.

\section{References}

1. Chadwick RG, M.Lindsay S (1996) Dental injuries during general anaesthesia. Br Dent J 180:255-258

2. Wang LP, Hagerdal M (1992) Reported anaesthetic complications during an 11-year period. A retrospective study. Acta Anaesthesiol Scand 36:234-240

3. Kaste LM, Gift HC, Swango PA (1996) Prevalence of incisor trauma in persons 6 to 50 years of age, United States, 1988-1991. J Dent Res 75:696-705

4. Burton JF, Baker AB (1987) Dental damage during anaesthesia and surgery. Anaesth Intens Care 15:262-268

5. Singleton RJ, Luddbrook GL, Webb RK, Fox MAL(1993) Physical injuries and environmental safety in anaesthesia: an analysis of 2000 incident reports. Anaesth Intens Care 21:659-663

6. Magnin C, Bory EN, Motin J (1991) Traumatismes dentaires au cours de I'intubation: un nouveau dispositif de prévention. Ann Fr Anaesth Réanim 10:171-174

7. Shigematsu T, Kobayashi M, Yorozu T, Takizawa K (1995) Use of new plastic teeth protector during endotracheal intubation. J Clin Anaesth 19:431-432 